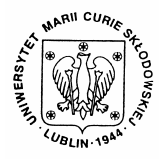

Annales UMCS Informatica AI 8(1) (2008) 177-184

$10.2478 / \mathrm{v} 10065-008-0017-1$

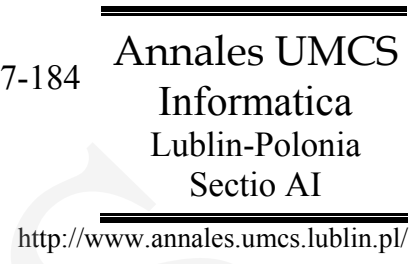

\title{
Interconnection distributed systems designed with hierarchical organization
}

\author{
Mirosław Hajder, Andrzej Paszkiewicz \\ Rzeszów University of Technology, Wincentego Pola 2, 35-959 Rzeszów, Poland
}

\begin{abstract}
In this paper an origin approach to distributed systems design is shown. It allows to include complete costs of their life circuit. In order to simplify complexity of a design procedure we assume that a created system will have a regional hierarchical structure. A model based on the equivalence relation can describe that problem. In this way, it is possible to describe hierarchical regionalization simply for $\mathrm{n}$-levels.
\end{abstract}

\section{Introduction}

Designing, building and exploitation of the diffused information systems is a very complicated matter. First of all, its complexity is a result of the large, geographical dispersion, abundance of elements and functional variety of tasks.

The best way to solve most problems in these systems is using multi-channel and topological dualism [1].

But widening of the projection of new tasks concerning the structure and the reconfiguration of logical sources functioning on the base of unchanging physical components that might be realized using present methods, can exceed designer's abilities. To avoid this situation, this work presents a new organization of connections of the distributed system, called hierarchical regionalization, which will be realized on the basis of multi-channel available in wireless and optical communicative media. Thanks to allocating the level of logical combinations off, the offered regionalization has information but not geographical, character.

It means that the users employing the same information resources, can act in the same virtual network that is not connected with their locations. The hierarchical regionalization assures the adaptation of the system connection architecture to the user's needs and allows the system to lengthen its "life".

\footnotetext{
${ }^{*}$ Corresponding author: e-mail address: \{mhajder; andrzejp\}@prz.rzeszow.pl
} 
There are many popular ways of designing network connections of the diffused systems [2]. But most of them can not be used to design hierarchical networks based on multi-channel and topological dualism. Most of them are used to create one of the elements from dual topology (physical or logical network) and do not take significant limitations of designing the second dual element into account. This designing is accomplished on the topological basis and does not take into account functioning of information system, such as: lack of possibilities of limiting the numbers of physical or logical channels which are accessible in every connection; costs of the project does not consider the values of regenerating and hardening elements; also broadcasting and group motion are not taken into an account. It is assumed that there are no qualitative and quantitative limitations in the building nodes of connecting network. We find it necessary to change the existing methods of designing such networks and elaborate new ones.

\section{Algebraic system model with hierarchical regionalization}

In order to describe hierarchical regionalization formally we will consider a set $A=\left\{a_{1}, a_{2}, \ldots a_{n}\right\}$ that consists of the finite number of elements. Elements of the set $A$ have types $\tau_{1}, \tau_{2}, \ldots, \tau_{m}$ that are linearly sorted i.e. $\tau_{1}<\tau_{2}<\ldots<\tau_{m}$. These types of the set elements $A$ allow for include the equivalence relation $R$ into the set $A$.

$$
\forall a, b \in A \quad a R b \Leftrightarrow \tau(a)=\tau(b) .
$$

The equivalence relation $R$ allows to include the set of equivalence classes $A / R=\left\{A_{1}, A_{2}, \ldots, A_{m}\right\}$, where $A_{i}$ - class of the elements of only one type, $i=1,2, \ldots, m$. Linear order relation on types put elements of the set $A / R$ in order linearly, $A_{1}<A_{2}<\ldots<A_{n}$ more exactly, where $A_{i}<A_{j}$ means that $\tau_{i}(a)<\tau_{j}(b)$, $a \in A_{i}, b \in A_{j}, i, j=1,2, \ldots, m, i \neq j$.

But including a relation into the classes is insufficient. This ordering needs to be moved to the elements of set $A$. In this case we will introduce one more relation with the help of density function $f: A \rightarrow \mathbb{N}$, where $\mathbb{N}$ - the set of counting numbers. The density function $f: A \rightarrow \mathbb{N}$ amounts to the following: $f(a)=c$, where $c \in \mathbb{N}$ determines the number of elements $b$ type $\tau$, that comply with the element $a$ and $\tau(a)<\tau(b)$. Moreover, $f(a)=0$ if $a \in A_{1}$ and $f(a) \geq 0$ with $a \in A_{j}, j=2,3, \ldots, m$.

The density function $f$ allows introducing a derived relation $\bar{R}$ for the equivalence relation $R[10]$ :

$$
a \bar{R} b \Leftrightarrow a R b \quad \text { and } \quad f(a)=f(b)
$$


for all $a, b \in A$. It is obvious that the relation $\bar{R}$ is also an equivalence and then the set of equivalence classes $A / \bar{R}$ can be shown as:

$$
A / \bar{R}=\left\{A_{11}, A_{12}, \ldots, A_{1 k_{1}}, A_{21}, \ldots, A_{2 k_{2}}, A_{m 1}, \ldots, A_{m k_{m}}\right\}
$$

where:

$$
A_{i}=\bigcup_{j=1}^{k_{i}} A_{i j}, i=1,2, \ldots, m .
$$

This model allows describing hierarchical regionalization effectively and is used to create interconnection systems. Distinct from previous methods it can also show direct connections between any levels of the hierarchical region links.

\section{Mathematical definition of the design problem}

Let us assume that the distributed teleinformation system has hierarchical regional organization. The analyzed system has two levels and consists of a backbone and an access network to which the nodes of end users are directly connected. The set of nodes of end users is described as $V_{A}=\{1,2, \ldots, n\}$. The backbone nodes will be allocated together with the selected nodes of end users. The set of $V_{B}=\{1,2, \ldots, m\}$ includes the nodes in which it is possible to place the nodes of system backbone, $V_{B} \subseteq V_{A}$. There are also the nodes in the system in which the backbone node must be located. They are elements of the set $V_{C}=\{1,2, \ldots, k\}$.

Let a binary variable describe a connection between nodes. Let us assume that $i$ determines the end user node and $j$ is the node of backbone system. The existence of direct connection between $i$ and $j$ determines the binary variable $b_{i j}$, whose value is described by the following expression:

$$
b_{i j}= \begin{cases}1 & \text { if } i \text { is connected with } j, \\ 0 & \text { otherwise. }\end{cases}
$$

We assume that computer resources that end users are located only in the nodes backbone system. The intensity of using resources teleinformation system will be described as intensity of demands access to resources. We assume that demands reported by users are homogeneous. Let us separate them into two levels. The demands of the first level are served in the backbone node, to which the user node is connected. The demands of the second level are addressed to the resources of backbone node. Access to this node requires using the network backbone. Demands in both levels determine end users's demand for information processing. Efficiency of computational resources that are arranged in the root node will be determined by means of a number of served demands. We introduce the following symbols for the first level serving demands: $\varpi_{i}^{i n}-$ demands intensity of $i$-th end user that are addressed to own information 
resources; $\varpi_{i}^{\text {out }}$ - intensity of reply to demands of $i$-th end user access to own information resources; $\varpi_{i}$ - intensity of service demands of $i$-th access user to own resources. For the intensity of service demands the following relationship is used: $\varpi_{i}=\varpi_{i}^{i n}+\varpi_{i}^{\text {out }}$. Service demands of the second level are described in the following way: $\vartheta_{i s}^{i n}$ - intensity of appearance access demands to resources of $s$-th end user by the service task of $i$-th end user, $s, i \in V_{A}, \vartheta_{i s}^{\text {out }}$ - intensity of reply to service demands; $\vartheta_{i s}$ - intensity of demands service of $i$-th access user to $s$-th user's resources. For the second level demands, the following relationship is used: $\vartheta_{i s}=\vartheta_{i s}^{\text {in }}+\vartheta_{i s}^{\text {out }}$. The efficiency of computational node is determined as $\beta^{*}$.

Let us consider the cost of physical communication connections realization and suppose that it will be proportional to their length. The length $d_{i j}$ of the physical linking channel between $i$-th and $j$-th nodes $\left(i, j \in V_{A}\right)$ is determined on the basis of length orthodrome model [11]:

$$
d_{i j}=\arccos \left[\sin \left(\varphi_{i}\right) \sin \left(\varphi_{j}\right)+\cos \left(\varphi_{i}\right) \cos \left(\varphi_{j}\right) \cos \left(\alpha_{i}-\alpha_{j}\right)\right],
$$

where: $\left(\alpha_{i}, \varphi_{i}\right),\left(\alpha_{j}, \varphi_{j}\right)$ - geographical coordinates of nodes $i, j ; \alpha, \varphi-$ geographical length and width respectively. The length of the channel determined by expression is characterized by a significant mistake that can be minimalized by converting the model to the following:

$$
d_{i j}=2 \arcsin \left[\sqrt{\sin \left[\frac{\left(\varphi_{i}-\varphi_{j}\right)}{2}\right]^{2}+\cos \left(\varphi_{i}\right) \cos \left(\varphi_{j}\right) \sin \left[\frac{\left(\alpha_{i}-\alpha_{j}\right)}{2}\right]^{2}}\right] .
$$

Expression (7) is used for estimating real costs of communication physical channel building. In this case the following symbols will be introduced: $d_{i j}-$ length of channel that connects nodes $i$ and $j ; c^{r e g}-$ cost of signal optical regenerator; $c^{w z m}-$ cost of optical signal amplifier; $c^{\text {reg }}$ - unit cost of fiber channel; fiber $f_{i j}$ - a number of fibres between the nodes $i$ and $j ; c_{i j}$ - constant cost of communication; $\gamma$ - regeneration factor, i.e. frequency of distribution regenerator; $\delta$ - amplification factor i.e. frequency of amplifier distributing; $c_{i j}^{i o}$, - cost of sending-receiving. The cost of physical communication channel can be expressed by:

$$
k_{i j}=d_{i j}\left(f_{i j} c^{s w}+c_{i j}\right)+c^{r e g} \frac{d_{i j}}{\gamma}+c^{w z m} \frac{d_{i j}}{\delta}+f_{i j} c_{i j} .
$$

Costs of building physical communication channels were included in the square matrix $K=\left\|k_{i j}\right\|$, whose diagonal elements are equal to zero (i.e. $k_{i i}=0$ ). 
This matrix is symmetrical to diagonal twills and determines adding costs of $i$-th node to the resources arranged in $j$-th node. The set $V_{B}^{i} \subseteq V_{B}$ includes a subset of end users node set. It is likely to join these nodes to $i$-th end user.

The number $f_{i j}$ of fibers (or virtual communication channels) connecting i-th end user with $j$-th core node is estimated from the expression: $\left.f_{i j}=\right] \varpi_{i} t^{*} / \mu \rho_{i j} t^{*}\left[\right.$, where: $t^{*}-$ maximum total delay transmission of demand and reply: $\rho_{i j}$ - effective capacity of communication channel $i j$ in bits per second; $\mu$-average length of demands and replies in bits.

A second important element of system building costs are expenditures for fitting core nodes with hardware that processes service demands. Let us assume that costs of creating computational resources are proportional to an intensity of process demands stream. The following expression is introduced: $\omega-$ an intensity of process demands stream; $\Gamma^{W}(\omega)$ - constant cost function of building and exploitation of process node with the demand efficiency $\omega$ in time unit; $\Gamma_{j k}^{R}(\omega)$ - constant function building and operating backbone channel that connects nodes $j, k \in V_{B}$ for total demand intensity $\omega$ in both directions [6,7].

In view of the above considerations we can accept that the design objective function can be described by:

$$
C=\min \left[\sum_{j \in V_{B}} \sum_{i \in V_{A}} k_{i j} b_{i j}+\sum_{j \in V_{B}} \Gamma^{W}\left(\beta_{j}\right)+\sum_{j \in V_{B}} \sum_{\substack{k \in V_{B} \\ k>j}} \Gamma_{j k}^{R}\left(\sum_{\substack{i \in V_{A} \\ s \neq i}} \sum_{\substack{s \in A \\ s \neq i}}\left(\vartheta_{i s}+\vartheta_{s i}\right) b_{i j} b_{s k}\right)\right],
$$

where: $\beta_{j}$ - corresponds to processing capacity for nodes, in which the allocation of backbone nodes is acceptable and the following constraints must be pointed out:

$$
\sum_{j \in V_{B}^{i}} b_{i j}=1, \forall i \in V_{A},
$$

it guarantees, that any end user will be connected to its backbone node

$$
\sum_{i \in V_{A}} b_{i j} \geq 1, \forall j \in V_{C},
$$

and to any each node $j \in V_{C}$ at least one end user should be connected. Otherwise, it is not necessary that $j \in V_{C}$;

$$
\begin{gathered}
\beta_{j} \leq \beta^{*}, \forall j \in V_{B}, \\
\beta_{j}=\sum_{i \in V_{A}}\left(\varpi_{i}^{i n}+\Theta_{i}^{i n}\right) b_{i j},
\end{gathered}
$$

where: $\Theta_{i}^{i n}=\sum_{\substack{k \in V_{A} \\ k \neq i}} \vartheta_{i k}^{i n}$ 
Let $V_{D}=\{1,2, \ldots, l\}$ presents the set of the communication nodes in the accessible network. When we specify the accessible network as a dendrite we should include some restrictions for an objective function.

$$
\begin{gathered}
\sum_{j \in V_{A}} \vartheta_{j i}^{\text {in }}-\sum_{j \in V_{A}} \vartheta_{i j}^{\text {out }} \geq 0 \quad \forall i \in V_{D} \\
\sum_{j \in V_{A}} \vartheta_{j i}^{\text {in }}-\sum_{j \in V_{A}} \vartheta_{i j}^{\text {out }}=0 \quad \forall i \in V_{A} \backslash V_{D} \\
\vartheta_{j i} \leq \mathrm{M} z_{i j} \quad \forall(i, j) \in V_{A}
\end{gathered}
$$

where $f_{i j}$ is the flow on the link $(i, j)$, and $M$ means a huge constant.

\section{Research}

The above assumptions are the basis for designing the multileveled hierarchical connecting structure. In our research we used the full searching method HFS, where we consider the whole $\mathrm{V}_{\mathrm{A}}$ as a class of potential elements $\mathrm{V}_{\mathrm{b}}$. This method checks all possible combinations that is why it is timeconsuming and also uses many ways of calculation. As we wanted to improve this method, we decided to change the HFS2 version and took into account not only qualitative but also location limitations of possible locations of the backbone nodes. This procedure allows us to shorten the time of searching the solution and also limit the sublevel of possible solutions. However, there are some location limitations in reality. It means that some elements from the $\mathrm{V}_{\mathrm{A}}$ set are not possible elements from the $\mathrm{V}_{\mathrm{B}}$ set.

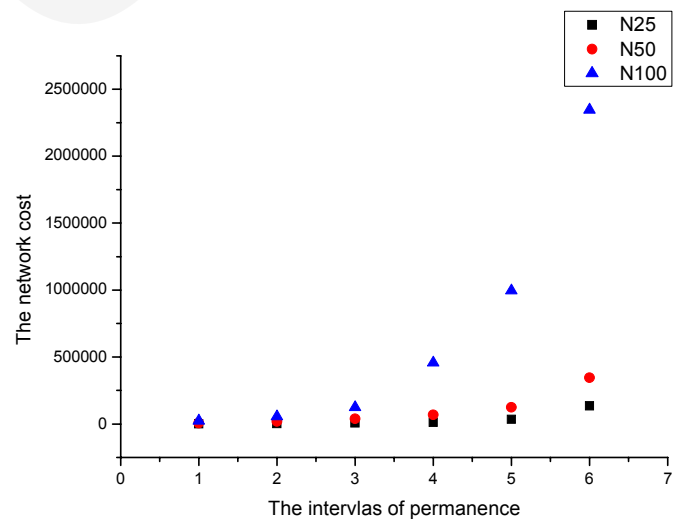

Fig. 1. The total network cost for $\mathrm{N}=25,50$ and 100 with the change of interval of capacity takes into account ( $N$ - number of nodes)

The above diagram shows the changes of network cost that is varied from the interval of its nodes capacity. To make this simulation clearer, we choose only one interval of capacity for all backbone nodes every time. To define some 
nodes and network we used the algorithm of maximum covering. In this algorithm where we had to define the optimal weight of some areas, we used a copy of priorities of activity and development areas.

The diagram below shows searching of solution where the selection of the first element was used.

The other diagram shows the situation, where the weight of the candidate (point) cannot be smaller than the product 0.85 coefficient and the weight of the best candidate. The results show improvement with the increasing iterations.
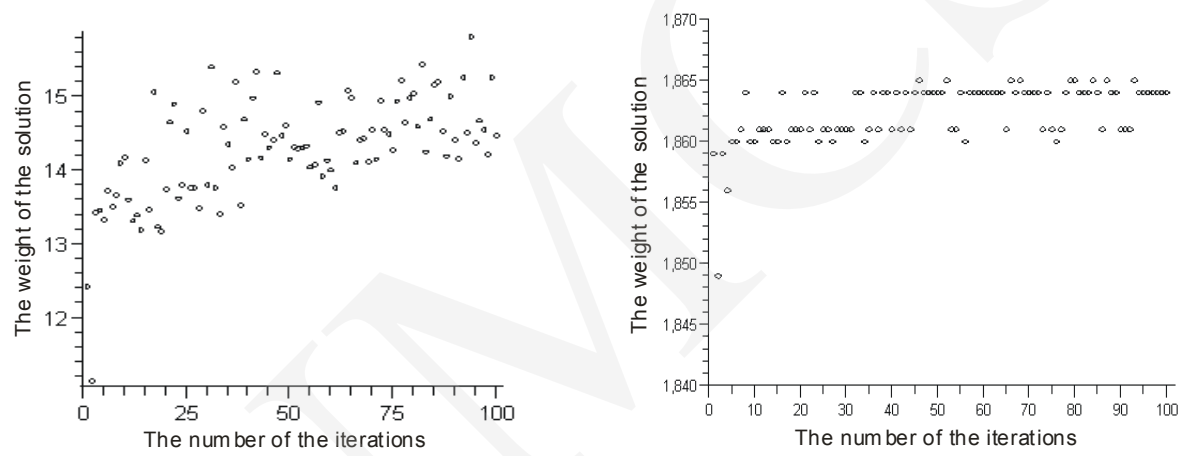

Fig. 2 The total weight of all chosen areas of the backbone nodes and accessible network localization: a) with total random selection of the initial elements (candidates) set,

b) with selection of the initial elements (candidates) set with 0.85 probability of selection of the highest possible weight

\section{Conclusion}

Designing hierarchical distributed systems is a very important area in a range of working calculating-connecting structures. Therefore, it is also important to create proper methods for their description. We also need to create algorithms of nodes localization and connections between them.

This suggested method for describing regional hierarchical structures allows referring to their elements, and also shows, how easy this solution is. Every single element of the system is identified in an explicit way with regard to other (superior or secondary) system elements.

However if you want to be satisfied with your result, you have to make a right choice of backbone nodes localization and nodes of the accessible network.

\section{References}

[1] Hartman D.H., Optical Interconnection Technology in the Telecommunications Network. Bell Communication Research, Morristown, New Jersey, (1986).

[2] Spohn D.L., Data Network Design. Second Edition. McGraw-Hill, New York, (1997).

[3] Kostrikin A.I., Wstep do algebry. Podstawy algebry. Wydawnictwa Naukowe PWN, Warszawa, (2004), (in Polish). 
Pobrane z czasopisma Annales AI- Informatica http://ai.annales.umcs.pl

Data: 26/04/2023 09:43:22

[4] Wróbel F., Nawigacja morska - zadania z objaśnieniami. Trademark; 2006; ISBN: 83915444-8-6.

[5] Hajder M., Dymora P., Mazurek M., Projektowanie topologii transparentnych sieci optycznych. Konferencja: Polski Internet Optyczny: technologie, usługi i aplikacje; Poznań, (2002) 183, (in Polish).

[6] Hajder M., Bolanowski M., Paszkiewicz A., Design of Improvement Efficiency of MultiChannel Optical Communication System Criteria Optimization. Annales Universitatis Mariae Curie-Skłodowska - Informatica. Lublin, 1 (2003) 271. 